\title{
Rational guidewire use in the coronary chronic total occlusion interventions
}

\author{
Ahmet Karabulut ${ }^{12^{*}}$ D, Sevket Gorgulu ${ }^{3}$ and Tanıl Kocagoz ${ }^{2}$
}

\begin{abstract}
Background: Procedures for coronary chronic total occlusion (CTO) are still a clinical challenge with relatively lower success rates. Recent advances in the biotechnology and introduction of CTO-dedicated guidewires have increased the procedural success rate of CTO interventions. Herein, we aimed to reveal the clinical and angiographic predictors of the crossability of the initial guidewire choice and rational guidewire usage in CTO interventions. A total of 177 patients with an indication for a coronary CTO procedure were included in this study. The use of 1-3 guidewires and crossing of the CTO lesion with the initial guidewire choice was defined as rational guidewire usage. The CTO lesions were classified according to the Japanese chronic total occlusion registry (J-CTO) and EuroCTO scores for evaluating the difficulty of the procedures. Then, a statistical analysis was performed to assess the initial guidewire choice, crossability, and contributors to rational guidewire usage.

Results: The mean J-CTO score was $1.42 \pm 1.16$, and the mean EuroCTO score was $1.44 \pm 1.18$. The success rate of the procedures was $90.4 \%$. The initial guidewire choice crossed the lesion in $44.1 \%$ of the cases, in which 1-3 guidewires were used (82.1\%). The crossability of the polymeric and moderate stiff tip guidewires was higher $(82.1 \%$ and $64.1 \%$, respectively), and the Pilot series was the most successful brand (36.2\%). Logistic regression analysis confirmed that JСTO score, procedural technique, guidewire type, and stiffness of the tip were the major predictors of rational guidewire usage.

Conclusion: Our analysis showed that the use of polymeric and moderate stiff tip guidewires, particularly the Pilot brand, were associated with rational guidewire usage in easy and intermediate difficulty CTO cases.

Keywords: Affordability, Coronary chronic total occlusion, Clinical practice, Guidewires, Percutaneous coronary interventions
\end{abstract}

\section{Background}

Chronic total occlusions (CTO) are one of the most challenging targets of percutaneous coronary interventions (PCI). Compared to non-CTO PCI, the procedural success rate is lower, and it has greater complication rates, more radiation exposure, and longer procedural times due to its complexity $[1,2]$.

\footnotetext{
* Correspondence: drkarabulut@yahoo.com

'Department of Cardiology, Acibadem Mehmet Ali Aydınlar University School of Medicine, Acibadem Atakent Hospital,Turgut Ozal Bulvarı, No: 16, 34303 Istanbul, Turkey

${ }^{2}$ Department of Medical Biotechnology, Acibadem Mehmet Ali Aydınlar University School of Medicine, Istanbul, Turkey

Full list of author information is available at the end of the article
}

The presence of CTO was the strongest independent predictor of incomplete PCI in patients with multivessel diseases, which is associated with a worse prognosis [2]. With the conventional PCI strategy, the success rate of CTO interventions is relatively lower compared to standard coronary lesions [3]. The success rate can be as low as $50 \%$, especially in older lesions with previous intervention. The cornerstone manoeuver in CTO interventions is the penetration of the CTO segment with a 0.014 inch guidewire [4-6]. This step is crucial for successful CTO interventions, and this step mainly depends on the choice of guidewire. There have been recent brilliant innovations in the dedicated guidewires for CTO lesions [7-10]. However, the release of new technologic guidewires does not 
mean the optimal use of new technology in clinical practice under real-world circumstances. Although newly introduced guidewires are smarter and facilitate guidewire penetration, their clinical usage usually varies according to the operator and clinical center. Availability, higher cost, and routine practice of the operator are the major determinants of guidewire preference. The guidelines usually do not define optimal guidewire usage, and the opinions of experts are usually the main factor in the preference of coronary guidewires.

Herein, we aimed to investigate the crossability and rational use of coronary guidewires in CTO interventions. We also aimed to assess the availability and preference of newly released technologic guidewires. We also discussed the clinical and angiographic determinants of the crossability of the initial guidewire choice and its clinical implications.

\section{Methods}

\section{Patient selection}

The investigation is a multicenter, cross-sectional, retrospective-prospective, and observational analysis of subsequent coronary CTO procedures. Between January 1, 2019, and April 30, 2020, a total of 177 patients with an indication for a coronary CTO procedure were included in this study. All the coronary CTO procedures included in this study were performed by the same CTO team. Since our primary endpoint was the evaluation of the biomaterials used in the procedure, a retrospective analysis of the cases with incomplete recordings and lack of clinical and laboratory data were excluded from this study.

\section{Protocol and procedure}

Upon admission, patients were evaluated with anamnesis and physical examination. Then, blood samples were taken for laboratory analysis. Echocardiography was performed before the procedure, and hypokinesia in the territory of the CTO lesion was assessed in detail. Images of each CTO procedure were evaluated, and the Japanese chronic total occlusion registry (J-CTO) score and EuroCTO score of each lesion were calculated according to previously defined criteria [11]. All the biomaterials, including catheters, guidewires, balloons, and stent materials, were analyzed extensively. The preferred techniques in the procedure were also recorded. The number of guidewires, initial choice of guidewire, and final guidewire that crossed the lesion were noted. The amount of contrast, duration of the procedure, and duration of fluoroscopy were also recorded for each procedure. Determinants of the crossability of the initial guidewire choice were evaluated with further statistical analysis. The selection of guidewires was made according to lesion characteristics, availability of the guidewire brands, and preference of the operator.

\section{Definition}

CTO was defined as total occlusion of one major epicardial coronary vessel of at least 3 months duration or undetermined time. The complexity of the CTO lesion and difficulty of the procedure were analyzed according to the J-CTO and EuroCTO scores. A score of 0 was accepted as an easy case, a score of 1 was defined as an intermediate case, a score of 2 was defined as a difficult case, and a score $\geq 3$ was accepted as a very difficult case.

Guidewires were classified according to their coatings and tip stiffness. A tip weight load $<1 \mathrm{~g}$ was accepted as a soft guidewire, while a tip weight load $\geq 9 \mathrm{~g}$ was defined as a stiff guidewire. With regard to tip coatings, the guidewires were classified as polymeric or non-polymeric. The number of 0.014 inch guidewires used was classified as optimal [1-3], acceptable [4-6], overuse [7-9], and overmuch $(\geq 10)$.

Simple crossing of the CTO segment was not defined as a successful procedure. Stent implantation with a distal thrombolysis in myocardial infarction (TIMI) flow of 2-3 was defined as a successful CTO procedure.

\section{Statistical analysis}

Statistical analyses were performed using SPSS 21.0 software (SPSS Inc., Armonk, NY, USA). Mean \pm standard deviation, median, and maximum-minimum were used for continuous variables, while percentages were used for categorical variables. Student's $t$ test and one-way ANOVA test were used for evaluating continuous variables between groups. Categorical variables were compared using the chi-square test and Fisher's exact test. Preference for the guidewires was analyzed according to the procedural technique and the J-CTO and EuroCTO scores. Pearson and Spearman correlation analyses were performed to define the determinants of crossability of the initial guidewire choice. Finally, univariate and multivariate regression analyses were performed to analyze the independent predictors of crossability of the initial guidewire choice. The baseline variables with evident significance $(p<$ 0.10 ) found by univariate analysis were included in the multivariate logistic regression analysis. The results of the model were reported as $95 \%$ confidence intervals (CIs) and $p$ values. All $p$ values were two-sided in the tests, and $p$ values $<0.05$ were considered to be statistically significant.

\section{Ethical concern}

This study was approved by the Ethics Committee of the Acibadem Mehmet Ali Aydinlar University (date: December 29, 2019; decision number: 2019-20/22).

\section{Results}

The baseline demographic and clinical characteristics of the patients are summarized in Table 1 . The procedural success rate was $91.4 \%$. The lesion could not be crossed 
Table 1 Baseline demographic characteristics, clinical features, and laboratory results of the patients. Angiographic, technical, and procedural features of chronic total occlusion lesions were also summarized in the lower part of the table

\begin{tabular}{|c|c|c|c|}
\hline Variables & $\%(n: 177)$ & Variables & $\%(n: 177)$ \\
\hline Age (years) & $62.4 \pm 10.5$ & Stable angina pectoris & $75.7 \%(134)$ \\
\hline Range & $29-86$ years & CCS class 1 & $4.5 \%(8)$ \\
\hline Sex & & CCS class 2 & $31.6 \%(56)$ \\
\hline Male & $79.7 \%(141)$ & CCS class 3 & $35.6 \%(63)$ \\
\hline Female & $20.3 \%(36)$ & CCS class 4 & $4 \%(7)$ \\
\hline Presence of diabetes mellitus & $37.3 \%(57)$ & Unstable angina & $4.5 \%(8)$ \\
\hline Presence of hypertension & $54.2 \%(96)$ & Previous Ml & $29.3 \%(52)$ \\
\hline Presence of dyslipidemia & $43.5 \%(77)$ & Previous CABG & $18.1 \%(32)$ \\
\hline Smoking & & Documentation of ischemia & $29.9 \%(53)$ \\
\hline Current smoker & $32.2 \%(57)$ & LV EF & \\
\hline Ex smoker & $20.3 \%(36)$ & $>50 \%$ & $66.7 \%(118)$ \\
\hline BMI $\left(\mathrm{kg} / \mathrm{m}^{2}\right)$ & $28.27 \pm 4.42$ & $35-50 \%$ & $28.8 \%(51)$ \\
\hline Initial creatinine (mg/dl) & $1.00 \pm 0.33$ & $<35 \%$ & $4.5 \%(8)$ \\
\hline Post-procedural creatinine (mg/dl) & $1.04 \pm 0.34$ & Hypokinesia in cto territory & $50.3 \%(89)$ \\
\hline Initial GFR (ml/minutes) & $90.3 \pm 31.8$ & Akinesia in cto territory & $2.3 \%(4)$ \\
\hline Vessel & & J-CTO Score & $1.42 \pm 1.16$ \\
\hline LAD & $32.8 \%(58)$ & 0 & $26.0 \%(46)$ \\
\hline LCX & $19.8 \%(35)$ & 1 & $28.2 \%(50)$ \\
\hline RCA & $47.5 \%(84)$ & 2 & $27.7 \%(49)$ \\
\hline Segment & & 3 & $13.6 \%(24)$ \\
\hline Ostial & $6.8 \%(12)$ & 4 & $4.0 \%(7)$ \\
\hline Proximal & $36.7 \%(65)$ & 5 & $0.6 \%(1)$ \\
\hline Mid & $46.3 \%(82)$ & Euroscore & $1.44 \pm 1.18$ \\
\hline Distal & $10.2 \%(18)$ & 0 & $23.2 \%(41)$ \\
\hline In-stent occlusion & $13 \%(23)$ & 1 & $31.1 \%(55)$ \\
\hline Multilevel occlusion & $11.9 \%(21)$ & 2 & $32.8 \%(58)$ \\
\hline Bifurcation in cto segment & $33.9 \%(60)$ & 3 & $6.8 \%(12)$ \\
\hline Radial access & $58.8 \%(104)$ & 4 & $4.0 \%(7)$ \\
\hline Contra-lateral injection & $53.1 \%(94)$ & 5 & $1.7 \%(3)$ \\
\hline Retrograde approach & $4 \%(7)$ & 6 & $0.6 \%(1)$ \\
\hline Guiding catheter & & Procedural technique & \\
\hline $6 \mathrm{~F}$ & $16.9 \%(30)$ & Single-wire cross & $60.5 \%(107)$ \\
\hline $7 F$ & $83.1 \%(147)$ & Parallel-wire cross & $6.2 \%(11)$ \\
\hline \multirow[t]{3}{*}{ Successfull procedure } & $90.4 \%(170)$ & Step up—step down & $23.2 \%(41)$ \\
\hline & & Dissection and re-entry & $8.5 \%(15)$ \\
\hline & & Reversed cart & $1.7 \%(3)$ \\
\hline
\end{tabular}

BMI body mass index, GFR glomerular filtration rate, CCS Canadian Cardiovascular Society, MI myocardial infarction, CABG coronary artery bypass grafting, LV EF left ventricular ejection fraction, $L A D$ left anterior descending artery, $L C X$ left circumflex artery, $R C A$ right coronary artery, $F$ French, $J$-CTO Japan registry of chronic total occlusion

in 17 cases, which were recorded as failed procedures. The mean age of patients was $62.4 \pm 10.5$ years, and 141 patients $(79.7 \%)$ were male. The prevalence of HT and DM were $54.2 \%$ and $37.3 \%$, respectively. Nearly $30 \%$ of the cases had an anamnesis significant for previous MI. A previous history of CABG was reported in $18.1 \%$ of the cases. Approximately three-fourths of the patients presented with stable angina pectoris, and half of the patients showed segmental left ventricular wall hypokinesia.

Angiographic and procedural features of the CTO lesions are also summarized in Table 1 . The right 
coronary artery was the most common vessel having a CTO segment $(47.5 \%)$. The middle and proximal segmental locations were more common compared to the distal and ostial locations. The lesion characteristics including bifurcation, in-stent occlusion, multivessel occlusion that aggravated the difficulty of the procedure was $<50 \%$. The J-CTO and EuroCTO scores revealed that $55 \%$ of the lesions were of either easy or intermediate difficulty, and the number of very difficult cases was definitely low (18.2\% of the cases). Single-wire crossing came to the forefront as a major procedural technique, followed by the step up-step down strategy $(60.5 \%$ and $23.2 \%$, respectively).

Guidewire choice and their characteristics are summarized in Table 2. The average number of guidewires used was $4.32 \pm 2.39$, and in $47.5 \%$ of the cases, $1-3$ guidewires were used during the procedure, which was described as a rational use of guidewires. The initial guidewire choice was usually the polymeric type, and the Fielder and Pilot brands were the most commonly preferred guidewires $(26.6 \%$ and $36.2 \%$, respectively). Moderate and soft tip load guidewires were preferred over stiff tip guidewires as a first choice (moderate tip load: $55.4 \%$ and stiff tip load: $12.4 \%$ ). Stiff tip and non- polymeric guidewires were more frequently preferred as a final guidewire compared to the initial choice. The Pilot, Fielder, and Gaia brands were the most successful guidewires in terms of crossing the CTO segment. Although the Gaia brand was not a favorite for the initial guidewire choice, it was the final choice in $13 \%$ of the cases.

The crossability of the initial guidewires and their clinical correlation are summarized in Tables 3 and 4. Initially, the preferred guidewire crossed the lesion in 78 patients (44.1\%). Polymeric and moderate stiff tip guidewires had superior crossability. Pilot 200, Pilot 50, and Fielder XT had higher crossability rates as a first-choice guidewire $(30.8 \%, 20.5 \%$, and $20.5 \%$, respectively). Even within the same brand, there were opposing results. Contrary to the Fielder XT, the crossability of the Fielder FC was not good (1.3\% vs $7.1 \%$, respectively). This result might indicate that distal tip composition is crucial to crossability. Within the non-polymeric groups, Confianza 9 had a relatively higher likelihood of lesion penetration as an initial guidewire choice.

Table 5 reveals the impact of procedural strategy on the guidewire choice. The polymeric Pilot brands were the most common initial guidewire choice in the single-

Table 2 Type and structural features of 0.014 inch coronary guidewires used for chronic total occlusion procedure

\begin{tabular}{|c|c|c|c|}
\hline Variables & $\%(n: 177)$ & Variables & $\%(n: 177)$ \\
\hline Guidewire number & $4.32 \pm 2.39$ & Final guidewire & \\
\hline $1-3$ & $47.5 \%(84)$ & Fielder brand & $22.6 \%(40)$ \\
\hline $4-6$ & $36.7 \%(65)$ & Miracle brand & $5.1 \%(9)$ \\
\hline $7-10$ & $12.4 \%(22)$ & Pilot brand & $26.6 \%(47)$ \\
\hline$>10$ & $3.4 \%(6)$ & Gaia brand & $13 \%(23)$ \\
\hline Initial guide wire & & Confianza brand & $9 \%(16)$ \\
\hline Fielder brand & $26.6 \%(47)$ & Ultimate brand & $1.7 \%(3)$ \\
\hline Miracle brand & $6.2 \%(11)$ & Sion brand & $2.3 \%(4)$ \\
\hline Pilot brand & $36.2 \%(64)$ & PT brand & $1.7 \%(3)$ \\
\hline Gaia brand & $2.3 \%(4)$ & Gladius brand & $5.6 \%(10)$ \\
\hline Confianza brand & $11.3 \%(20)$ & Progress brand & $2.3 \%(4)$ \\
\hline Ultimate brand & $6.8 \%(12)$ & Cross-it brand & $0.6 \%(1)$ \\
\hline Sion brand & $5.6 \%(10)$ & Failed procedure & $9.6 \%(17)$ \\
\hline PT brand & $2.3 \%(4)$ & Final guidewire type & \\
\hline Gladius brand & $2.3 \%(4)$ & Polymeric & $56.5 \%(100)$ \\
\hline Progress brand & $0.5 \%(1)$ & Non-poylmeric & $33.9 \%(60)$ \\
\hline Initial guidewire type & $16.9 \%(30)$ & Final guidewire stiffness & \\
\hline Polymeric & $67.2 \%(119)$ & Soft (< 1 gram) & $24.9 \%(44)$ \\
\hline Non-poylmeric & $32.8 \%(58)$ & Moderate (1-9 g) & $47.5 \%(84)$ \\
\hline Initial guidewire stiffness & & Stiff ( $\geq 9 \mathrm{~g}$ ) & $18.1 \%(32)$ \\
\hline Soft $(<1 \mathrm{~g})$ & $32.2 \%(57)$ & Same initial_-final guidewire & $44.1 \%(78)$ \\
\hline Moderate (1-9 g) & $55.4 \%(98)$ & & \\
\hline Stiff ( $\geq 9 \mathrm{~g})$ & $12.4 \%(22)$ & & \\
\hline
\end{tabular}


Table 3 Clinical and angiographic predictors of first-choice guidewire and it's crossability through the CTO segment

\begin{tabular}{|c|c|c|c|}
\hline Variables & Same initial-final guidewire $(+)$ & Same initial-final guidewire (-) & $p$ \\
\hline Age (years) & $63.41 \pm 10.9$ & $61.74 \pm 10.2$ & 0.30 \\
\hline Sex & & & 0.07 \\
\hline Male & $71.8 \%(56)$ & $84.8 \%(84)$ & \\
\hline Female & $26.9 \%(21)$ & $15.2 \%(15)$ & \\
\hline $\mathrm{BMl}(\mathrm{mg} / \mathrm{dl})$ & $27.9 \pm 4.65$ & $28.5 \pm 4.23$ & 0.42 \\
\hline Initial creatinine (mg/dl) & $0.96 \pm 0.30$ & $1.04 \pm 0.36$ & 0.12 \\
\hline GFR (ml/minutes) & $64.2 \pm 22.6$ & $68.7 \pm 21.3$ & 0.76 \\
\hline LV EF & & & 0.07 \\
\hline$>50 \%$ & $73.1 \%(57)$ & $61.6 \%(61)$ & \\
\hline $35-50 \%$ & $20.5 \%(16)$ & $35.4 \%(35)$ & \\
\hline$<35 \%$ & $6.4 \%(5)$ & $3.0 \%(3)$ & \\
\hline Hypokinesia in cto territory & $41 \%(32)$ & $57.6 \%(57)$ & 0.09 \\
\hline Procedural technique & & & $<0.01$ \\
\hline Single-wire cross & $88.5 \%(69)$ & $38.4 \%(38)$ & \\
\hline Parallel wire cross & $1.3 \%(1)$ & $10.1 \%(10)$ & \\
\hline Step up—step down & $5.1 \%(4)$ & $37.4 \%(37)$ & \\
\hline Dissection and re-entry & $5.1 \%(4)$ & $11.1 \%(11)$ & \\
\hline Reversed cart & $0 \%(0)$ & $3 \%(3)$ & \\
\hline Radial access & $55.1 \%(43)$ & $61.6 \%(61)$ & 0.38 \\
\hline Kontralateral contrast injection & $48.7 \%(38)$ & $56.6 \%(56)$ & 0.29 \\
\hline J-CTO Score & $0.83 \pm 0,88$ & $1.89 \pm 1.13$ & $<0.01$ \\
\hline 0 & $42.3 \%(33)$ & $13.1 \%(13)$ & \\
\hline 1 & $37.2 \%(29)$ & $21.2 \%(21)$ & \\
\hline 2 & $16.7 \%(13)$ & $36.4 \%(36)$ & \\
\hline 3 & $2.6 \%(2)$ & $22.2 \%(22)$ & \\
\hline 4 & $1.3 \%(1)$ & $6.1 \%(6)$ & \\
\hline 5 & $0 \%(0)$ & $1 \%(1)$ & \\
\hline EuroCTO score & $0.93 \pm 0.90$ & $1.84 \pm 1.23$ & $<0.01$ \\
\hline Target vessel & & & 0.89 \\
\hline LAD & $34.6 \%(27)$ & $31.3 \%(31)$ & \\
\hline LCX & $19.2 \%(15)$ & $20.2 \%(20)$ & \\
\hline RCA & $46.2 \%(36)$ & $48.5 \%(48)$ & \\
\hline CTO segment & & & 0.50 \\
\hline Ostial & $5.1 \%(4)$ & $8.1 \%(8)$ & \\
\hline Proximal & $33.3 \%(26)$ & $39.4 \%(39)$ & \\
\hline Mid & $52.6 \%(41)$ & $41.4 \%(41)$ & \\
\hline Distal & $9 \%(7)$ & $11.1 \%(11)$ & \\
\hline Guidewire number & $2.85 \pm 1.31$ & $5.48 \pm 2.43$ & $<0.01$ \\
\hline Ballon number & $2.85 \pm 1.44$ & $3.20 \pm 1.88$ & 0.18 \\
\hline Stent number & $1.58 \pm 0.70$ & $1.91 \pm 0.91$ & 0.01 \\
\hline Stent length (mm) & $50.6 \pm 21.8$ & $65.7 \pm 33.0$ & 0.001 \\
\hline Fluoroscopy duration & $22.2 \pm 13.1$ & $45.6 \pm 24.4$ & $<0.01$ \\
\hline Contrast amount (ml) & $242 \pm 114$ & $362 \pm 173$ & $<0.01$ \\
\hline Total & $44.1 \%(78)$ & $55.9 \%(99)$ & \\
\hline
\end{tabular}

$B M I$ body mass index, GFR glomerular filtration rate, $L V E F$ left ventricular ejection fraction, J-CTO Japan registry of chronic total occlusion, $L A D$ left anterior descending artery, $L C X$ left circumflex artery, RCA right coronary arterY, CTO chronic total occlusion

$P<0.05$ is indicated as significant 
Table 4 Distributions of guidewire type, number, and brands according to crossability of initial choice guidewire

\begin{tabular}{|c|c|c|c|}
\hline Variables & Same initial-final guidewire (+) & Same initial-final guidewire (-) & $p$ \\
\hline Initial guidewire type & & & $<0.001$ \\
\hline Polymeric & $82.1 \%(64)$ & $55.6 \%(55)$ & \\
\hline Non-polymeric & $17.9 \%(14)$ & $44.4 \%(44)$ & \\
\hline Initial guidewire stiffness & & & 0.07 \\
\hline Soft $(<1 \mathrm{~g})$ & $28.2 \%(22)$ & $35.4 \%(35)$ & \\
\hline Moderate (1-9 g) & $64.1 \%(50)$ & $48.5 \%(48)$ & \\
\hline Stiff ( $\geq 9 \mathrm{~g}$ ) & $7.7 \%(6)$ & $16.2 \%(16)$ & \\
\hline Guidewire number & & & $<0.001$ \\
\hline $1-3$ & $82.1 \%(64)$ & $20.2 \%(20)$ & \\
\hline $4-6$ & $15.4 \%(12)$ & $53.5 \%(53)$ & \\
\hline $7-10$ & $2.6 \%(2)$ & $20.2 \%(20)$ & \\
\hline$\geq 10$ & $0 \%(0)$ & $3.4 \%(6)$ & \\
\hline Guidewire brand & & & 0.005 \\
\hline Fielder FC & $1.3 \%(1)$ & $7.1 \%(7)$ & \\
\hline Fielder XT & $20.5 \%(16)$ & $14.1 \%(14)$ & \\
\hline Fielder XT-A & $1.3 \%(1)$ & $3.0 \%(3)$ & \\
\hline Fielder XT-R & $5.1 \%(4)$ & $1.0 \%(1)$ & \\
\hline Miracle 3 & $1.3 \%(1)$ & $1.0 \%(1)$ & \\
\hline Miracle 6 & $3.8 \%(3)$ & $6.1 \%(6)$ & \\
\hline Miracle 12 & $0 \%(0)$ & $0 \%(0)$ & \\
\hline Pilot 50 & $20.5 \%(16)$ & $12.1 \%(12)$ & \\
\hline Pilot 150 & $1.3 \%(1)$ & $0 \%(0)$ & \\
\hline Pilot 200 & $30.8 \%(24)$ & $11.1 \%(11)$ & \\
\hline Gaia 1 & $0 \%(0)$ & $1.0 \%(1)$ & \\
\hline Gaia 2 & $1.3 \%(1)$ & $1.0 \%(1)$ & \\
\hline Gaia 3 & $0 \%(0)$ & $1.0 \%(1)$ & \\
\hline Confianza 9 & $7.7 \%(6)$ & $14.1 \%(14)$ & \\
\hline Sion & $1.3 \%(1)$ & $9.1 \%(9)$ & \\
\hline Sion black & $0 \%(0)$ & $0 \%(0)$ & \\
\hline Ultimate 3 & $2.6 \%(2)$ & $10.1 \%(10)$ & \\
\hline PT 2 & $0 \%(0)$ & $4.0 \%(4)$ & \\
\hline Gladius & $1.3 \%(1)$ & $3.0 \%$ (3) & \\
\hline Progress 200 & $0 \%(0)$ & $1.0 \%(1)$ & \\
\hline Total & $44.1 \%(78)$ & $55.9 \%(99)$ & \\
\hline
\end{tabular}

$P<0.05$ is indicated as significant

wire cross technique (41.1\%). The polymeric Fielder brands were more commonly preferred for the parallel wire technique $(45.5 \%)$. The non-polymeric Gaia and Confianza brands were preferred in the step up-step down strategy and in the dissection and re-entry technique. Soft guidewires were the dominant choice for the retrograde approach.

The correlation between procedural difficulty and guidewire choice is revealed in Tables 6 and 7 in terms of CTO score. Both the J-CTO and EuroCTO scores showed similar correlations with guidewire preference and performance. Polymer jacketed guidewires were most commonly used in lesions with CTO scores of 0 1 , whereas non-polymeric guidewires were most commonly used in lesions with CTO scores of 3-4. The polymeric Pilot and Fielder brand guidewires were the most common initial guidewire choices. The Confianza brand was the most commonly used non-polymeric guidewire in lesions with CTO scores of 2-4. The average number of guidewires used was also statistically 
Table 5 Distribution of first-choice and final crossing guidewires characteristics according to a procedural technique

\begin{tabular}{|c|c|c|c|c|c|c|}
\hline Variables & Single wire & Parallel wire & Step up Step down & Dissection re-entry & Reversed cart & $p$ \\
\hline Guidewire number & $3.63 \pm 2.23$ & $4.27 \pm 1.19$ & $5.36 \pm 2.26$ & $5.80 \pm 2.56$ & $7.66 \pm 2.08$ & $<0.001$ \\
\hline $1-3$ & $65.4 \%(70)$ & $36.4 \%(4)$ & $19.5 \%(8)$ & $13.3 \%(2)$ & $0 \%(0)$ & \\
\hline $4-6$ & $24.3 \%(26)$ & $63.6 \%(7)$ & $53.7 \%(22)$ & $60.0 \%(9)$ & $33.3 \%(1)$ & \\
\hline $7-10$ & $7.5 \%(8)$ & $0 \%(0)$ & $24.4 \%(10)$ & $20.0 \%(3)$ & $33.3 \%(1)$ & \\
\hline$>10$ & $2.8 \%(3)$ & $0 \%(0)$ & $2.4 \%(1)$ & $6.7 \%(1)$ & $33.3 \%(1)$ & \\
\hline Initial guide wire & & & & & & 0.01 \\
\hline Fielder brand & $25.2 \%(27)$ & $45.5 \%(5)$ & $24.4 \%(10)$ & $26.7 \%(4)$ & $33.3 \%(1)$ & \\
\hline Miracle brand & $5.6 \%(6)$ & $9.1 \%(1)$ & $7.3 \%(3)$ & $6.7 \%(1)$ & $0 \%(0)$ & \\
\hline Pilot brand & $41.1 \%(44)$ & $27.3 \%(3)$ & $31.7 \%(13)$ & $26.7 \%(4)$ & $0 \%(0)$ & \\
\hline Gaia brand & $0.9 \%(1)$ & $0 \%(0)$ & $2.4 \%(1)$ & $13.3 . \%(2)$ & $0 \%(0)$ & \\
\hline Confianza brand & $5.6 \%(6)$ & $9.1 \%(1)$ & $22.0 \%(9)$ & $26.7 \%(4)$ & $0 \%(0)$ & \\
\hline Ultimate brand & $7.5 \%(8)$ & $9.1 \%(1)$ & $7.3 \%(3)$ & $0 \%(0)$ & $0 \%(0)$ & \\
\hline Sion brand & $8.4 \%(9)$ & $0 \%(0)$ & $0 \%(0)$ & $0 \%(0)$ & $33.3 \%(1)$ & \\
\hline PT brand & $2.8 \%(3)$ & $0 \%(0)$ & $0 \%(0)$ & $0 \%(0)$ & $33.3 \%(1)$ & \\
\hline Gladius brand & $1.9 \%(2)$ & $0 \%(0)$ & $4.9 \%(2)$ & $0 \%(0)$ & $0 \%(0)$ & \\
\hline Progress brand & $0.9 \%(1)$ & $0 \%(0)$ & $0 \%(0)$ & $0 \%(0)$ & $0 \%(0)$ & \\
\hline Initial guidewire type & & & & & & 0.57 \\
\hline Polymeric & $71.0 \%(76)$ & $72.7 \%(8)$ & $61.0 \%(25)$ & $53.3 \%(8)$ & $66.7 \%(2)$ & \\
\hline Non-poylmeric & $29.0 \%(731)$ & $27.3 \%(3)$ & $39.0 \%(16)$ & $46.7 \%(7)$ & $33.3 \%(1)$ & \\
\hline Initial wire stiffness & & & & & & 0.01 \\
\hline Soft $(<1 \mathrm{~g})$ & $35.5 \%(38)$ & $36.4 \%(4)$ & $19.5 \%(8)$ & $26.7 \%(4)$ & $100 \%(3)$ & \\
\hline Moderate (1-9g) & $57.9 \%(62)$ & $54.5 \%(6)$ & $58.5 \%(24)$ & $40.0 \%(6)$ & $0 \%(0)$ & \\
\hline Stiff ( $\geq 9 \mathrm{~g})$ & $6.5 \%(7)$ & $9.1 \%(1)$ & $22.0 \%(9)$ & $33.3 \%(5)$ & $0 \%(0)$ & \\
\hline Final guidewire & & & & & & $<0.001$ \\
\hline Fielder brand & $29.3 \%(27)$ & $0 \%(0)$ & $12.5 \%(5)$ & $57.1 \%(8)$ & $0 \%(0)$ & \\
\hline Miracle brand & $6.5 \%(6)$ & $9.1 \%(1)$ & $5.0 \%(2)$ & $0 \%(0)$ & $0 \%(0)$ & \\
\hline Pilot brand & $40.2 \%(37)$ & $9.1 \%(1)$ & $15.0 \%(6)$ & $21.4 \%(3)$ & $0 \%(0)$ & \\
\hline Gaia brand & $10.9 \%(10)$ & $27.3 \%(3)$ & $22.5 \%(9)$ & $0 \%(0)$ & $33.3 \%(1)$ & \\
\hline Confianza brand & $7.6 \%(7)$ & $18.2 \%(2)$ & $17.5 \%(7)$ & $0 \%(0)$ & $0 \%(0)$ & \\
\hline Ultimate brand & $2.2 \%(2)$ & $0 \%(0)$ & $2.5 \%(1)$ & $0 \%(0)$ & $0 \%(0)$ & \\
\hline Sion brand & $1.1 \%(1)$ & $9.1 \%(1)$ & $5.0 \%(2)$ & $0 \%(0)$ & $0 \%(0)$ & \\
\hline PT brand & $0 \%(0)$ & $0 \%(0)$ & $0 \%(0)$ & $21.4 \%(3)$ & $0 \%(0)$ & \\
\hline Gladius brand & $1.9 \%(2)$ & $9.1 \%(1)$ & $12.2 \%(5)$ & $0 \%(0)$ & $66.7 \%(2)$ & \\
\hline Progress brand & $0 \%(0)$ & $18.2 \%(2)$ & $4.9 \%(2)$ & $0 \%(0)$ & $0 \%(0)$ & \\
\hline Cross-it brand & $0 \%(0)$ & $0 \%(0)$ & $2.4 \%(1)$ & $0 \%(0)$ & $0 \%(0)$ & \\
\hline Failed procedure & $14.0 \%(15)$ & $0 \%(0)$ & $2.4 \%(0)$ & $6.7 \%(1)$ & $0 \%(0)$ & \\
\hline Final guidewire type & & & & & & $<0.001$ \\
\hline Polymeric & $71.7 \%(66)$ & $18.2 \%(2)$ & $40.0 \%(16)$ & $100 \%(14)$ & $66.7 \%(2)$ & \\
\hline on-poylmeric & $28.3 \%(2)$ & $81.8 \%(9)$ & $60.0 \%(24)$ & $0 \%(0)$ & $33.3 \%(1)$ & \\
\hline Final wire stiffness & & & & & & 0.001 \\
\hline Soft $(<1 \mathrm{~g})$ & $29.3 \%(27)$ & $9.1 \%(1)$ & $17.5 \%(7)$ & $64.3 \%(9)$ & $0 \%(0)$ & \\
\hline Moderate (1-9g) & $57.6 \%(53)$ & $36.4 \%(4)$ & $50.0 \%(20)$ & $35.7 \%(5)$ & $66.7 \%(2)$ & \\
\hline Stiff $(\geq 9 \mathrm{~g})$ & $13.0 \%(12)$ & $54.5 \%(6)$ & $32.5 \%(13)$ & $0 \%(0)$ & $33.3 \%(1)$ & \\
\hline Same initial-final wire & $64.5 \%(69)$ & $9.1 \%(1)$ & $9.8 \%(4)$ & $26.7 \%(4)$ & $0 \%(0)$ & $<0.001$ \\
\hline Total & $60.5 \%(107)$ & $6.2 \%(11)$ & $23.2 \%(41)$ & $8.5 \%(15)$ & $1.7 \%(3)$ & \\
\hline
\end{tabular}


Table 6 Distribution of first-choice and final crossing guidewires characteristics according to J-CTO score

\begin{tabular}{|c|c|c|c|c|c|c|c|}
\hline Variables & J-СТО 0 & J-Сто 1 & J-СТО 2 & J-СTO 3 & J-Сто 4 & J-СТО 5 & $p$ \\
\hline Guidewire number & $3.21 \pm 1.57$ & $3.60 \pm 1.88$ & $4.87 \pm 2.50$ & $6.04 \pm 2.05$ & $7.28 \pm 4.02$ & $3.0 \pm 1.0$ & $<0.001$ \\
\hline $1-3$ & $73.9 \%(34)$ & $60.0 \%(30)$ & $32.7 \%(16)$ & $8.3 \%(2)$ & $14.3 \%(1)$ & $100 \%(1)$ & \\
\hline $4-6$ & $21.7 \%(10)$ & $30.0 \%(15)$ & $49.0 \%(24)$ & $54.2 \%(13)$ & $42.9 \%(3)$ & $0 \%(0)$ & \\
\hline $7-10$ & $4.3 \%(2)$ & $8.0 \%(4)$ & $14.3 \%(7)$ & $33.3 \%(8)$ & $14.3 \%(1)$ & $0 \%(0)$ & \\
\hline$>10$ & $0 \%(0)$ & $2.0 \%(1)$ & $4.1 \%(2)$ & $4.2 \%(1)$ & $28.6 \%(2)$ & $0 \%(0)$ & \\
\hline Initial guide wire & & & & & & & 0.12 \\
\hline Fielder brand & $34.8 \%(16)$ & $24.0 \%(12)$ & $24.5 \%(12)$ & $29.2 \%(7)$ & $0 \%(0)$ & $0 \%(0)$ & \\
\hline Miracle brand & $4.3 \%(2)$ & $6.0 \%(3)$ & $8.2 \%(4)$ & $4.2 \%(1)$ & $14.3 \%(1)$ & $0 \%(0)$ & \\
\hline Pilot brand & $47.8 \%(22)$ & $44.0 \%(22)$ & $30.6 \%(1)$ & $12.5 \%(3)$ & $28.6 \%(2)$ & $0 \%(0)$ & \\
\hline Gaia brand & $0 \%(0)$ & $2.0 \%(1)$ & $2.0 \%(1)$ & 8.3.\% (2) & $0 \%(0)$ & $0 \%(0)$ & \\
\hline Confianza brand & $2.2 \%(1)$ & $6.0 \%(3)$ & $12.2 \%(6)$ & $29.2 \%(7)$ & $42.9 \%(3)$ & $0 \%(0)$ & \\
\hline Ultimate brand & $4.3 \%(2)$ & $4.0 \%(2)$ & $12.2 \%(6)$ & $8.3 \%(2)$ & $0 \%(0)$ & $0 \%(0)$ & \\
\hline Sion brand & $2.2 \%(1)$ & $6.0 \%(3)$ & $8.2 \%(4)$ & $4.2 \%(1)$ & $14.3 \%(1)$ & $0 \%(0)$ & \\
\hline PT brand & $0 \%(0)$ & $4.0 \%(2)$ & $0 \%(0)$ & $4.2 \%(1)$ & $0 \%(0)$ & $100 \%(1)$ & \\
\hline Gladius brand & $2.2 \%(1)$ & $4.0 \%(2)$ & $2.0 \%(1)$ & $0 \%(0)$ & $0 \%(0)$ & $0 \%(0)$ & \\
\hline Progress brand & $2.2 \%(1)$ & $0 \%(0)$ & $0 \%(0)$ & $0 \%(0)$ & $0 \%(0)$ & $0 \%(0)$ & \\
\hline Initial guidewire type & & & & & & & 0.001 \\
\hline Polymeric & $84.8 \%(39)$ & $76.0 \%(38)$ & $57.1 \%(28)$ & $45.8 \%(11)$ & $28.6 \%(2)$ & $100 \%(1)$ & \\
\hline Non-poylmeric & $15.2 \%(7)$ & $24.0 \%(12)$ & $42.9 \%(21)$ & $54.2 \%(13)$ & $71.4 \%(5)$ & $0 \%(0)$ & \\
\hline Initial guidewire stiffness & & & & & & & 0.02 \\
\hline Soft $(<1 \mathrm{~g})$ & $34.8 \%(16)$ & $32.0 \%(16)$ & $30.6 \%(15)$ & $33.3 \%(8)$ & $14.3 \%(1)$ & $100 \%(1)$ & \\
\hline Moderate (1-9g) & $60.9 \%(28)$ & $62.0 \%(31)$ & $55.1 \%(27)$ & $37.5 \%(9)$ & $42.9 \%(3)$ & $0 \%(0)$ & \\
\hline Stiff ( $\geq 9 \mathrm{~g}$ ) & $4.3 \%(2)$ & $6.0 \%(3)$ & $14.3 \%(7)$ & $29.2 \%(7)$ & $42.9 \%(3)$ & $0 \%(0)$ & \\
\hline Final guidewire & & & & & & & 0.04 \\
\hline Fielder brand & $32.6 \%(15)$ & $17 \%(8)$ & $23.8 \%(10)$ & $31.6 \%(6)$ & $16.7 \%(1)$ & $0 \%(0)$ & \\
\hline Miracle brand & $4.3 \%(2)$ & $8.5 \%(4)$ & $2.4 \%(1)$ & $10.5 \%(2)$ & $0 \%(0)$ & $0 \%(0)$ & \\
\hline Pilot brand & $43.5 \%(20)$ & $38.3 \%(18)$ & $14.3 \%(6)$ & $10.5 \%(2)$ & $16.7 \%(1)$ & $0 \%(0)$ & \\
\hline Gaia brand & $8.7 \%(4)$ & $12.8 \%(6)$ & $21.4 \%(9)$ & $15.8 \%(3)$ & $16.7 \%(1)$ & $0 \%(0)$ & \\
\hline Confianza brand & $6.5 \%(3)$ & $12.8 \%(6)$ & $11.9 \%(5)$ & $10.5 \%(2)$ & $0 \%(0)$ & $0 \%(0)$ & \\
\hline Ultimate brand & $4.3 \%(2)$ & $0 \%(0)$ & $2.1 \%(1)$ & $0 \%(0)$ & $0 \%(0)$ & $0 \%(0)$ & \\
\hline Sion brand & $0 \%(0)$ & $2.1 \%(1)$ & $4.8 \%(2)$ & $5.3 \%(1)$ & $0 \%(0)$ & $0 \%(0)$ & \\
\hline PT brand & $0 \%(0)$ & $0 \%(0)$ & $4.8 \%(2)$ & $5.3 \%(1)$ & $0 \%(0)$ & $0 \%(0)$ & \\
\hline Gladius brand & $0 \%(0)$ & $6.0 \%(3)$ & $6.1 \%(3)$ & $8.3 \%(2)$ & $28.6 \%(2)$ & $0 \%(0)$ & \\
\hline Progress brand & $0 \%(0)$ & $2.0 \%(1)$ & $4.1 \%(2)$ & $0 \%(0)$ & $14.3 \%(1)$ & $0 \%(0)$ & \\
\hline Cross-it brand & $0 \%(0)$ & $0 \%(0)$ & $2.0 \%(1)$ & $0 \%(0)$ & $0 \%(0)$ & $0 \%(0)$ & \\
\hline Failed procedure & $0 \%(0)$ & $6.0 \%(3)$ & $14.3 \%(7)$ & $20.8 \%(5)$ & $14.3 \%(1)$ & $100 \%(1)$ & \\
\hline Final guidewire type & & & & & & & 0.15 \\
\hline Polymeric & $76.1 \%(35)$ & $61.7 \%(29)$ & $50.0 \%(21)$ & $57.9 \%(11)$ & $66.7 \%(4)$ & $0 \%(0)$ & \\
\hline Non-poylmeric & $23.9 \%(11)$ & $38.3 \%(18)$ & $50.0 \%(21)$ & $42.1 \%(8)$ & $33.3 \%(2)$ & $0 \%(0)$ & \\
\hline Final guidewire stiffness & & & & & & & 0.23 \\
\hline Soft $(<1 \mathrm{~g})$ & $30.4 \%(14)$ & $21.3 \%(10)$ & $28.6 \%(12)$ & $36.8 \%(7)$ & $16.7 \%(1)$ & $0 \%(0)$ & \\
\hline Moderate (1-9g) & $63.0 \%(29)$ & $55.3 \%(26)$ & $42.9 \%(18)$ & $42.1 \%(8)$ & $50.0 \%(2)$ & $0 \%(0)$ & \\
\hline Stiff ( $\geq 9 \mathrm{~g})$ & $6.5 \%(3)$ & $23.4 \%(11)$ & $28.6 \%(12)$ & $21.1 \%(4)$ & $33.3 \%(2)$ & $0 \%(0)$ & \\
\hline Same initial-final wire & $71.7 \%(33)$ & $58 \%(29)$ & $26.5 \%(13)$ & $8.3 \%(2)$ & $14.3 \%(1)$ & $0 \%(0)$ & $<0.001$ \\
\hline Total & $26.0 \%(46)$ & $28.2 \%(50)$ & $27.7 \%(49)$ & $13.6 \%(24)$ & $4.0 \%(7)$ & $0.6 \%(1)$ & \\
\hline
\end{tabular}

$J$-CTO Japan registry of chronic total occlusion, $g$ gram $P<0.05$ is indicated as significant 
Table 7 Distribution of first-choice and final crossing guidewire characteristics according to EuroCTO score

\begin{tabular}{|c|c|c|c|c|c|c|c|}
\hline Variables & E-СТО 0 & E-СTO 1 & Е-СТО 2 & Е-СТО 3 & Е-СТО 4 & Е-СТO 5 & $p$ \\
\hline Guidewire number & $3.29 \pm 1.79$ & $3.61 \pm 1.70$ & $5.31 \pm 2.57$ & $4.66 \pm 3.02$ & $5.42 \pm 2.43$ & $7.33 \pm 4.16$ & $<0.001$ \\
\hline $1-3$ & $70.7 \%(29)$ & $60.0 \%(33)$ & $27.6 \%(16)$ & $41.7 \%(5)$ & $14.3 \%(1)$ & $0 \%(0)$ & \\
\hline $4-6$ & $22.0 \%(9)$ & $30.9 \%(17)$ & $46.6 \%(27)$ & $41.7 \%(5)$ & $71.4 \%(5)$ & $66.7 \%(2)$ & \\
\hline $7-10$ & $7.3 \%(3)$ & $9.1 \%(5)$ & $20.7 \%(12)$ & $8.3 \%(1)$ & $0 \%(0)$ & $0 \%(0)$ & \\
\hline$>10$ & $0 \%(0)$ & $0 \%(0)$ & $5.2 \%(3)$ & $8.3 \%(1)$ & $14.3 \%(1)$ & $33.3 \%(1)$ & \\
\hline Initial guide wire & & & & & & & 0.06 \\
\hline Fielder brand & $31.7 \%(13)$ & $21.8 \%(12)$ & $25.9 \%(15)$ & $41.7 \%(5)$ & $28.6 \%(2)$ & $0 \%(0)$ & \\
\hline Miracle brand & $4.9 \%(2)$ & $5.5 \%(3)$ & $6.9 \%(4)$ & $8.3 \%(1)$ & $0 \%(0)$ & $33.3 \%(1)$ & \\
\hline Pilot brand & $48.8 \%(20)$ & $50.9 \%(28)$ & $22.4 \%(13)$ & $8.3 \%(1)$ & $28.6 \%(2)$ & $0 \%(0)$ & \\
\hline Gaia brand & $2.4 \%(1)$ & $1.8 \%(1)$ & $3.4 \%(2)$ & $0 \%(0)$ & $0 \%(0)$ & $0 \%(0)$ & \\
\hline Confianza brand & $2.4 \%(1)$ & $1.8 \%(1)$ & $22.4 \%(13)$ & $16.7 \%(2)$ & $14.3 \%(1)$ & $33.3 \%(1)$ & \\
\hline Ultimate brand & $2.4 \%(1)$ & $5.5 \%(3)$ & $10.3 \%(6)$ & $8.3 \%(1)$ & $14.3 \%(1)$ & $0 \%(0)$ & \\
\hline Sion brand & $4.9 \%(2)$ & $5.5 \%(3)$ & $6.9 \%(4)$ & $0 \%(0)$ & $0 \%(0)$ & $33.3 \%(1)$ & \\
\hline PT brand & $0 \%(0)$ & $1.8 \%(1)$ & $1.7 \%(1)$ & $8.3 \%(1)$ & $14.3 \%(1)$ & $0 \%(0)$ & \\
\hline Gladius brand & $0 \%(0)$ & $5.5 \%(3)$ & $0 \%(0)$ & $8.3 \%(1)$ & $0 \%(0)$ & $0 \%(0)$ & \\
\hline Progress brand & $2.4 \%(1)$ & $0 \%(0)$ & $0 \%(0)$ & $0 \%(0)$ & $0 \%(0)$ & $0 \%(0)$ & \\
\hline Initial wire type & & & & & & & 0.001 \\
\hline Polymeric & $80.5 \%(33)$ & $80.0 \%(44)$ & $50.0 \%(29)$ & $66.7 \%(8)$ & $71.4 \%(5)$ & $0 \%(0)$ & \\
\hline Non-poylmeric & $19.5 \%(8)$ & $20.0 \%(11)$ & $50.0 \%(29)$ & $33.3 \%(4)$ & $28.6 \%(2)$ & $100 \%(3)$ & \\
\hline Initial wire stiffness & & & & & & & 0.01 \\
\hline Soft $(<1 \mathrm{~g})$ & $34.1 \%(14)$ & $25.5 \%(14)$ & $32.8 \%(19)$ & $50.0 \%(6)$ & $42.9 \%(3)$ & $33.3 \%(1)$ & \\
\hline Moderate1-9 g) & $61.0 \%(25)$ & $70.9 \%(39)$ & $44.8 \%(26)$ & $33.3 \%(4)$ & $42.9 \%(3)$ & $33.3 \%(1)$ & \\
\hline Stiff ( $\geq 9 \mathrm{~g}$ ) & $4.9 \%(2)$ & $3.6 \%(2)$ & $22.4 \%(13)$ & $16.7 \%(2)$ & $14.3 \%(1)$ & $33.3 \%(1)$ & \\
\hline Final guidewire & & & & & & & 0.39 \\
\hline Fielder brand & $26.8 \%(11)$ & $19.6 \%(10)$ & $26.9 \%(14)$ & $37.5 \%$ (3) & $20.0 \%(1)$ & $0 \%(0)$ & \\
\hline Miracle brand & $7.3 \%(3)$ & $3.9 \%(2)$ & $5.8 \%(3)$ & $0 \%(0)$ & $20 \%(1)$ & $0 \%(0)$ & \\
\hline Pilot brand & $43.9 \%(18)$ & $39.2 \%(20)$ & $15.4 \%(8)$ & $12.5 \%(1)$ & $0 \%(0)$ & $0 \%(0)$ & \\
\hline Gaia brand & $12.2 \%(5)$ & $9.8 \%(5)$ & $21.2 \%(11)$ & $12.5 \%(1)$ & $0 \%(0)$ & $0 \%(0)$ & \\
\hline Confianza brand & $7.3 \%(3)$ & $13.7 \%(7)$ & $7.7 \%(4)$ & $12.5 \%(1)$ & $20.0 \%(1)$ & $0 \%(0)$ & \\
\hline Ultimate brand & $2.4 \%(1)$ & $3.9 \%(2)$ & $0 \%(0)$ & $0 \%(0)$ & $0 \%(0)$ & $0 \%(0)$ & \\
\hline Sion brand & $0 \%(0)$ & $2.0 \%(1)$ & $5.8 \%(3)$ & $0 \%(0)$ & $0 \%(0)$ & $0 \%(0)$ & \\
\hline PT brand & $0 \%(0)$ & $2.0 \%(1)$ & $5.8 \%(3)$ & $0 \%(0)$ & $0 \%(0)$ & $0 \%(0)$ & \\
\hline Gladius brand & $0 \%(0)$ & $3.6 \%(2)$ & $6.9 \%(4)$ & $16.7 \%(2)$ & $14.3 \%(1)$ & $33.3 \%(1)$ & \\
\hline Progress brand & $0 \%(0)$ & $1.8 \%(1)$ & $3.4 \%(2)$ & $0 \%(0)$ & $14.3 \%(1)$ & $0 \%(0)$ & \\
\hline Cross-it brand & $0 \%(0)$ & $0 \%(0)$ & $1.7 \%(1)$ & $0 \%(0)$ & $14.3 \%(1)$ & $0 \%(0)$ & \\
\hline Failed procedure & $0 \%(0)$ & $7.3 \%(4)$ & $10.3 \%(6)$ & $33.3 \%(4)$ & $28.6 \%(2)$ & $33.3 \%(1)$ & \\
\hline Final guidewire type & & & & & & & 0.50 \\
\hline Polymeric & $70.7 \%(29)$ & $64.7 \%(33)$ & $53.8 \%(28)$ & $75.0 \%(6)$ & $40.0 \%(2)$ & $50.0 \%(1)$ & \\
\hline Non-poylmeric & $29.3 \%(12)$ & $35.3 \%(18)$ & $46.2 \%(24)$ & $25.0 \%(2)$ & $60.0 \%(3)$ & $50.0 \%(1)$ & \\
\hline Final wire stiffness & & & & & & & 0.20 \\
\hline Soft $(<1 \mathrm{~g})$ & $24.4 \%(10)$ & $19.6 \%(10)$ & $36.5 \%(19)$ & $37.5 \%(3)$ & $20.0 \%(1)$ & $0 \%(0)$ & \\
\hline Moderate (1-9g) & $65.9 \%(27)$ & $60.8 \%(31)$ & $38.5 \%(20)$ & $37.5 \%(3)$ & $40.0 \%(2)$ & $50.0 \%(1)$ & \\
\hline Stiff $(\geq 9 \mathrm{~g})$ & $9.8 \%(4)$ & $19.6 \%(10)$ & $25.0 \%(13)$ & $25.0 \%(2)$ & $40.0 \%(2)$ & $50.0 \%(1)$ & \\
\hline Same initial-final wire & $68.3 \%(28)$ & $58.2 \%(32)$ & $24.1 \%(14)$ & $25.0 \%(3)$ & $14.3 \%(1)$ & $0 \%(0)$ & $<0.001$ \\
\hline Total & $23.2 \%(41)$ & $31.1 \%(55)$ & $32.8 \%(58)$ & $6.8 \%(12)$ & $4.0 \%(7)$ & $1.7 \%(3)$ & \\
\hline
\end{tabular}

EuroCTO 6 group: just 1 patient, guidewire number 8, initial wire is non-polymeric, stiff Confianza 9 brand, final wire is polymeric, soft Fielder FC brand, E-CTO Euro chronic total occlusion

$P<0.05$ is indicated as significant 
significant in correlation with both the J-CTO and EuroCTO scores. There was a linear correlation between the J-CTO score, EuroCTO score, and guidewire number. More complex cases were associated with a higher number of guidewires $(p<0.001)$. The final guidewire type and stiffness did not differ statistically. However, the polymeric Pilot brands were most successful in lesions with CTO scores of $0-1$. The non-polymeric Gaia brand had superior crossability in lesions with CTO scores of $2-4(p=0.04)$. The crossability of the initial guidewire choice had a close relationship with both the J-CTO and EuroCTO scores. The same initial and final guidewire was used in $71.7 \%$ of lesions with a J-CTO score of 0 , which dropped to $14.7 \%$ in lesions with a J-CTO score of 4 ( $p<0.001)$. This analysis showed that the crossability of the initial guidewires was higher in lesions with JCTO scores of $0-1$ and gradually declined as the score increased.

\section{Multivariate analysis}

Univariate and multivariate logistic regression analyses were performed to obtain determinants of the crossability of the initial guidewire. The procedural technique and J-CTO score were independent predictors of the crossability of the initial guidewire (OR 0.326, CI 0.199; $0.535, p<0.001$ and OR 0.363 , CI $0.240 ; 0.550, p<$ 0.001) (Table 8). A lower J-CTO score was a predictor of the use of fewer guidewires. The initial guidewire type and stiffness were also independent predictors of the success of the initial guidewire (OR 5.763, CI 1.980; 16.775, $p=0.001$ and OR 0.324, CI 0.149; 0.705, $p=$ $0.004)$. The initial guidewire brand did not have a significant impact on the crossability of the initial guidewire choice $(p=0.26)$.

Table 8 Multivariate logistic regression analysis to reveal the determinants of the crossability of initial choice guidewire

\begin{tabular}{llll}
\hline $\begin{array}{l}\text { Dependant variable: } \\
\text { Same initial-final } \\
\text { guidewire }\end{array}$ & Odds Ratio & \multicolumn{2}{l}{ Multivariate analysis } \\
\cline { 3 - 4 } & $\mathbf{9 5 \%} \mathbf{C l}$ & $\boldsymbol{p}$ \\
\hline Sex (male/female) & 0.312 & $0.112 ; 0.869$ & 0.02 \\
LV EF & 0.717 & $0.314 ; 1.640$ & 0.43 \\
Hypokinesia on cto territory & 0.733 & $0.342 ; 1.570$ & 0.42 \\
J-CTO Score & 0.363 & $0.240 ; 0.550$ & $<0.001$ \\
Procedural technique & 0.326 & $0.199 ; 0.535$ & $<0.001$ \\
Initial guidewire & 0.949 & $0.866 ; 1.040$ & 0.26 \\
Initial guidewire type & 5.763 & $1.980 ; 16.775$ & 0.001 \\
Initial guidewire stiffness & 0.324 & $0.149 ; 0.705$ & 0.004 \\
\hline
\end{tabular}

LV EF left ventricular ejection fraction, J-СTO Japan registry of chronic total occlusion

$P<0.05$ is indicated as significant

\section{Discussion}

This study was the first report from Turkey that analyzed rational guidewire use and procedural success in CTO procedures. We found that rational guidewire usage (1-3 guidewires) was possible, especially in easy and moderately difficult CTO procedures. The crossing of the CTO lesion with the initial guidewire choice decreased both biomaterial use and the cost of the procedure. Classification of the CTO lesion by the J-CTO or EuroCTO score led to a more precise strategy. Polymeric jacketed moderate stiff tip guidewires, particularly the Pilot brand, had superior crossability in the easy and moderately difficult CTO procedures.

Cardiac CTO procedures necessitate special instruments to increase the success rate. Recent advances in medical biotechnology have led to the manufacture of smarter coronary guidewires, which directly affect the procedural success of CTO interventions [12-15]. The availability of new generation CTO-specific biomaterials is still a concern in most cardiac centers. Most centers use a few brands of CTO-specific materials, including guidewires, microcatheters, and balloon catheters. Moreover, patients have to pay for a certain percentage of the CTO procedure by themselves because of the limited coverage of insurance companies. So, the rational use of such biomaterials is important for sustainable CTO interventions.

Each CTO-dedicated guidewire has a different tip structure, polymer jacket, and tip stiffness [14]. Variations in guidewire structure affect the steerability, crossability, and tactile feedback of the guidewire. Operators should be familiar with the structure of the guidewires, then they should form a strategy for the technique and guidewire choice [13]. The polymer-jacketed guidewire has superior steerability; however, a subintimal course is common, and due to limited tactile feedback, operators should use them very carefully $[7,16]$. Non-polymeric guidewires have a better tactile feedback and intraluminal course. However, their steerability is inferior to polymeric guidewires. A stiff tip is important for lesion penetration and wire escalation. A tough stump necessitates more stiff guidewires. Our experience shows that non-polymeric and stiff guidewires are more commonly preferred in complex lesions. Polymeric guidewires are more useful in easy and intermediate difficulty lesions. The presence of microchannels is the optimal indication for soft and moderate stiff tip polymeric guidewires [17-19].

Lesion characteristics including length, calcification, tortuosity, stump, distal area, and occlusion duration are all important for defining an optimal strategy $[18,19]$. Several CTO scores were proposed to determine the success of a CTO procedure [11]. Instead of evaluating the lesion characteristics one by one, simply totalling the CTO scores may predict the success rate of the procedure. The J-CTO 
score was developed with the help of the Japan Multicentre CTO registry in 2006. It was approved by investigations that predicted the success rate of antegrade CTO interventions [20]. A lower J-CTO score is usually accepted as an easy or intermediate difficulty case. Higher scores indicate very difficult cases. Our procedures were performed mostly by the antegrade approach. Thus, the JCTO score is a suitable method to assess our cases. Approximately $80 \%$ of the patients had lesions with J-CTO scores of $0-2$. So, we can conclude that our results are appropriate to analyze easy, intermediate, and difficult cases. Very difficult cases constituted just $17 \%$ of the total cases. There was an inverse correlation between the J-CTO score and success rate, which was in accord with the medical literature $[20,21]$. All lesions with a J-CTO of 0 had successful interventions. There was also an inverse relationship between the crossability of the initial guidewire choice and J-CTO score. More than 50\% of the lesions with J-CTO scores of $0-1$ were crossed with the initial guidewire. This statistic reveals the importance of the initial guidewire choice. Reasonable initial guidewire choice and the single-wire cross strategy would lead to shorter procedural and fluoroscopy times, as well as the use of fewer guidewires, balloons, and stents, which affect the affordability and long-term prognosis of the CTO procedure. Polymeric and moderate stiff tip guidewires showed a superior performance in crossing the CTO segment. The choice of the Pilot brand in lesions with J-CTO scores of $0-1$ would increase the probability of crossability with the initial guidewire. In such lesion types, non-polymeric stiff guidewires should not be used as the initial guidewire.

In each CTO procedure, the use of 1-3 guidewires can be accepted as an economical and rational use of guidewires. In each procedure, a standard soft tipped or polymer-jacketed guidewire was used to place the microcatheter. Then, special CTO guidewires replaced the initial standard wire for lesion penetration. In the final stage, the standard wire was exchanged with the CTO wire once again to perform the balloon and stenting procedure. Special CTO wire should be used just for lesion penetration. This strategy necessitates at least 2-3 wires for each procedure. In our cases, the average number of guidewires was $<4$ in lesions with J-CTO scores of $0-1$. We can conclude that the use of $>4$ guidewires with the antegrade technique in lesions with J-CTO scores of $0-1$ was not rational, and it can be defined as overuse. The crossability of the polymeric soft and moderate stiff tip guidewires was not so good in lesions with J-CTO scores $>2$. For this more complicated lesion, stiff tip nonpolymeric guidewires should be used. The Gaia brand showed a more significant crossability performance in lesions with J-CTO scores $>2$. The distribution of the Fielder brand was similar between all J-CTO scores. But there was a small detail in the performance of Fielder guidewires. In the lesions with J-CTO scores of $0-1$, the crossability of the Fielder guidewire as an initial choice was higher, and the single-wire technique was usually preferred. In the more complex cases, other techniques, particularly the step up and step down strategy, were preferred. Lesions were modified with multiple guidewires, including non-polymer stiff guidewires and Fielder guidewires, that were used to just jump to the true lumen. Thus, a similar distribution of the Fielder brand as a final guidewire did not indicate the strong crossability of lesions with J-CTO scores $>2$.

The EuroCTO or CASTLE score was defined with the help of the prospective EuroCTO registry [11]. This novel scoring chart was compared with the J-CTO score, and it was found to be superior in more complex cases. We performed a J-CTO score-like analysis also for the EuroCTO score. Our result was comparable for both scoring methods. Lesions with EuroCTO scores of $0-1$ showed a result similar to lesions with J-CTO scores of $0-1$. The average guidewire number was $<4$, and more than $50 \%$ of the lesions were crossed with the initial guidewire. Polymeric moderate stiff tip guidewires showed a superior performance in crossing the CTO segment as an initial guidewire choice. However, final wire brand, composition, and stiffness did not differ among the EuroCTO score group. Nonetheless, we can state that stiffer tip guidewires were preferred with higher EuroCTO scores. As a limitation, there were just four lesions with EuroCTO scores of 5-6, which significantly reduced the statistical analysis for very complex lesions. Non-polymeric moderate stiff tip guidewires were used more commonly in lesions with EuroCTO scores $1-4$ as a final guidewire compared to the initial wire type. Our data confirmed that the polymeric moderate stiff tip Pilot brand was a good choice for lesions with EuroCTO scores 0-1.

Our results strongly confirmed that the use of CTO scoring would influence our technique in a favorable way, which directly affect the initial guidewire choice. Single-wire escalation is associated with the use of fewer guidewires. For the easy and intermediate difficulty CTO lesions, single-wire crossing should be the initial choice for rational guidewire usage. Other techniques including parallel wire, step up-step down, and dissection and reentry were associated with a very low probability of penetration of the lesion with the initial guidewires. However, the parallel wire technique offered more rational guidewire use. The average number of guidewires used was lower when comparing to step up-step down and dissection and re-entry techniques. Polymeric guidewires were more successful in single-wire escalation. Non-polymeric guidewires should be chosen in the other techniques. According to lesion modification during wire escalation and drilling, optimal guidewire exchange 
should be performed. For the dissection and re-entry technique, polymeric guidewires should be preferred to jump into the true lumen.

The use of new generation guidewires would increase the success rate. However, the affordability of new generation guidewires would limit their optimal preference. The Fielder brand is one of the most commonly preferred soft polymeric guidewires. We usually preferred the Fielder XT series rather than the new generation Fielder XT-A. This choice may overshadow the crossability of the Fielder brand. The new generation Fielder XT-A series has an additional composite core technology in the guidewire tip, which enhances crossability. We used Fielder XT-A series in only four cases, which did not show statistically significant superiority. On the other hand, the new generation non-polymeric guidewires are preferred compared to the older series. The Miracle brand is a well-known nonpolymeric guidewire from Asahi Intecc. The new generation Gaia brand with composite core technology has replaced the Miracle series. In our experience, the Gaia brand was preferred over the Miracle series, which would directly affect the success rate. On the other hand, we used first-generation Gaia 1-2-3 brands. The newly released Gaia Next series, which has Xtrand coil technology, was not used in any of the cases. For more complex lesions that necessitate a stiff tip load, Confianza 9 was preferred over the Confianza Pro series, which possesses a thinner tip and has a more slippery hydrophilic coating. Moreover, we also used Gladius guidewires as a last resort in certain cases. The Gladius brand is a new generation peripheral guidewire, which has a durable balanced tip composition. It has relatively superior crossability for intermediate and difficult CTO lesions. Although it is designed for peripheral interventions, it can be used for coronary CTO procedures in selected cases (as a rescue solution). On the other hand, the newly released Gladius MG guidewire series was not used due to its unavailability.

Preference for new generation guidewires would lead to more successful procedural results [21, 22]. However, the experience of the operator was also just as important as technology [23]. Our case series, newly released guidewires were used in a few cases. Nonetheless, our procedural success rate was above the average (approximately $90 \%)$. Each operator has a special relationship with guidewires, and each has their favourite guidewire for different occasions. Experienced operators can predict the behavior of the guidewire in each of the different lesion compositions. Indeed, all operators want to perform CTO procedures with newly released technology. Unfortunately, it is not possible in most cardiac centres located in Turkey. For this reason, the operator should know the composition and behavior of all the guidewire brands to choose guidewires in a rational manner.

\section{Limitations}

The first limitation is a relatively small sample size. For assessing newly released biotechnology, we analyzed the recent past period of time. So, our case sample is relatively small. All the procedures were performed by the same CTO team, which raises a question about the personal preference of the guidewires. Although the procedures were performed by the same team, the cases were collected from different hospitals, which have their own catheter laboratory and independent purchasing department. So, the CTO operators preferred the guidewire according to its availability in the catheter laboratory.

\section{Conclusion}

Adequate strategy and logical guidewire choice are the key for successful CTO interventions. Although our success rate for the procedures was higher, the use of newly released guidewire technology was relatively limited. This result showed that it would take some time to get use to the newly released biomaterials in clinical practice. Classification of the CTO lesion by J-CTO and EuroCTO scores would influence the choice for strategy and guidewire preference. In lesions with J-CTO scores of $0-1$ and EuroCTO scores of $0-1$, the single-wire crossing technique was associated with the use of fewer guidewires. Most of these lesions could be crossed by the initial guidewires. Polymeric, moderate stiff tip guidewires, particularly the Pilot brand, had superior crossability as an initial guidewire. The same initial-final guidewire strategy also had fewer balloons used, shorter stent length, shorter fluoroscopy duration, and decreased amounts of contrast used. However, the same initial-final guidewire strategy was not successful in difficult and very difficult lesions (J-CTO score $>2$, EuroCTO score $>2$ ). In such groups, new innovative non-polymeric guidewires, particularly the Gaia brand, seemed to be superior with regard to lesion penetration.

\section{Abbreviations}

CTO: Chronic total occlusion; J-CTO: Japanese chronic total occlusion registry; PCl: Percutaneous coronary intervention; TIMI: Thrombolysis in myocardial infarction; SPSS: Statistical Package for the Social Sciences; Cl: Confidence interval; OR: Odds ratio; HT: Hypertension; DM: Diabetes mellitus;

MI: Myocardial infarction; CABG: Coronary artery bypass graft

\section{Acknowledgements \\ This study is derived from the doctorate thesis of Ahmet Karabulut entitled as "Preference and success rate of 0.014 inch guidewires in the coronary chronic total occlusion."}

\section{Authors' contributions}

AK: Hypothesis, data collection, writing, and the corresponding author. SG: Invasive procedure and data collection. TK: Literature review and final corrections. The authors have read and approved the manuscript.

\section{Funding}

There is no financial relationship with a company, and this work was not supported by any company. 


\section{Availability of data and materials}

The datasets used and/or analyzed during the current study are available from the corresponding author on reasonable request.

\section{Ethics approval and consent to participate}

This study was approved by the Ethics Committee of the Acibadem Mehmet Ali Aydinlar University (date: December 19, 2019; decision number: 2019-20/ 22). This investigation is a cross-sectional, retrospective-prospective, and observational study. Informed consent for invasive procedure was taken from all participants.

\section{Consent for publication}

Not applicable

\section{Competing interests}

The authors did not report any conflict of interest regarding this work.

\section{Author details}

'Department of Cardiology, Acibadem Mehmet Ali Aydınlar University School of Medicine, Acibadem Atakent Hospital,Turgut Ozal Bulvarı, No: 16, 34303 Istanbul, Turkey. ${ }^{2}$ Department of Medical Biotechnology, Acibadem Mehmet Ali Aydınlar University School of Medicine, Istanbul, Turkey. ${ }^{3}$ Department of Cardiology, Acibadem Kocaeli Hospital, Kocaeli, Turkey.

Received: 19 August 2020 Accepted: 23 October 2020

Published online: 07 November 2020

\section{References}

1. Sianos G, Konstantinidis NV, Di Mario C, Karvounis H (2016) Theory and proctical based approach to chronic total occlusions. BMC Cardiovasc Disord. https://doi.org/10.1186/s12872-016-0209-3

2. Karatasakis A, Danek BA, Karmpaliotis D et al (2017 Jan) Approach to CTO intervention: overview of techniques. Curr Treat Options Cardiovasc Med 19(1):1

3. Uzunlar B, Karabulut A, Dogan Z, Ozay B (2014) Ten-year patency of functionally expanded polytetrafluoroethylene coronary graft detected in an urgent cardiac surgery. Turk Gogus Kalp Dama 22(3):628-631

4. Erglis A (2010) Coronary guidewires. Eurointervention. 6:168-169

5. Buller CE (2013) Coronary guidewires for chronic total occlusion procedures: function and design. Interv Cardiol 5(5):533-540

6. Karatasakis A, Tarar MN, Karmpaliotis D et al (2017) Guidewire and microcatheter utilization patterns during antegrade wire escalation in chronic total occlusion percutaneous coronary intervention: insights from a contemporary multicenter registry. Catheter Cardiovasc Interv 89(4):E90-E98

7. Guidewires, In: Brilakis E editor. Manuel of chronic total occlusion interventions second edition; London, Elsevier, 2017: p 57-71.

8. Kandzari DE, Grantham JA, Karmpaliotis D et al (2018) Safety and efficacy of dedicated guidewire and microcatheter technology for chronic total coronary occlusion revascularization: principal results of the Asahilntecc chronic Total occlusion study. Coron Artery Dis 29(8):618-623

9. Khalili H, Vo MN, Brilakis ES (2016) Initial experience with the Gaia composite core guidewires in coronary chronic total occlusion crossing. J Invasive Cardiol 28(2):E22-E25

10. Vemmou E, Nikolakopoulos I, Xenogiannis I et al (2019) Recent advances in microcatheter technology for the treatment of chronic total occlusions. Expert Rev Med Devices 16(4):267-273

11. Kalogeropoulos AS, Alsanjari O, Keeble TR et al (2020) CASTLE score versus J-CTO score for the prediction of technical success in chronic total occlusion percutaneous revascularisation. Euro Intervention 15(18):e1615e1623

12. Karatasakis A, Brilakis ES (2019) Chronic total occlusion percutaneous coronary intervention failure: learning from failure. Catheter Cardiovasc Interv 93(6):1039-1040

13. Brilakis ES, Grantham JA, Rinfret $S$ et al (2012) A percutaneous treatment algorithm for crossing coronary chronic total occlusions. JACC CardiovascInterv 5(4):367-379

14. Dash D (2016) Guidewire crossing techniques in coronary chronic total occlusion intervention: a to Z. Indian Heart J 68(3):410-420

15. Kinnaird T, Gallagher S, Cockburn J et al (2018) Procedural success and outcomes with increasing use of enabling strategies for chronic total occlusion intervention. Circ Cardiovasc Interv 11(10):e006436
16. Mishra S (2016) Language of CTO interventions - focus on hardware. Indian Heart J 68(4):450-463

17. Wilson W, Spratt JC (2014 May) Advances in procedural techniquesantegrade. Curr Cardiol Rev 10(2):127-144

18. Tanaka H, Tsuchikane E, Muramatsu T et al (2019) A novel algorithm for treating chronic total coronary artery occlusion. J Am Coll Cardiol 74(19): 2392-2404

19. Brilakis ES, Mashayekhi K, Tsuchikane E et al (2019 Jul 30) Guiding principles for chronic total occlusion percutaneous coronary intervention. Circulation. 140(5):420-433

20. Nagamatsu W, Tsuchikane E, Oikawa Y et al. Predicting successful guidewire crossing via collateral channel at retrograde percutaneous coronary intervention for chronic total occlusion: the J-Channel score as a difficulty estimating tool for collateral channel guidewire crossing success from the Japanese CTO-PCI Expert Registry. Eurolntervention. 2019 Apr 23.

21. Galassi A, Grantham A Kandzari D et al. 2014 Percutaneous treatment of coronary chronic total occlusion part 2: technical approach. Interv Cardiol 9(3):201-207

22. Toth GG, Yamane M, Heyndricks GY (2015) How to select a guidewire: technical features and key characteristics. Heart 101:645-652

23. Young MN, Secemsky EA, Kaltenbach LA et al (2019 Aug) Examining the operator learning curve for percutaneous coronary intervention of chronic total occlusions. Circ Cardiovasc Interv. 12(8):e007877

\section{Publisher's Note}

Springer Nature remains neutral with regard to jurisdictional claims in published maps and institutional affiliations.

\section{Submit your manuscript to a SpringerOpen ${ }^{\circ}$ journal and benefit from:}

- Convenient online submission

- Rigorous peer review

- Open access: articles freely available online

High visibility within the field

- Retaining the copyright to your article

Submit your next manuscript at $\boldsymbol{\nabla}$ springeropen.com 